\title{
Cryo-EM Study of the Ribosome-Bound Release Factor 1
}

\author{
Urmila Rawat***, Haixiao Gao*, Andrey Zavialov***, Richard Gursky*, Mans \\ Ehrenberg***, and Joachim Frank ${ }^{* * * * * *}$ \\ * Howard Hughes Medical Institute, Health Research, Inc. at the Wadsworth Center, \\ Empire State Plaza, Albany, New York 12201-0509 \\ **National Institute of Diabetes and Digestive and Kidney Diseases, National Institute of \\ Health, Bethesda, MD 20892 (current address) \\ *** Department of Cell and Molecular Biology, Biomedical Center, S-75124 Uppsala, \\ Sweden. \\ ****Department of Biomedical Sciences, State University of New York at Albany, \\ Albany, New York, 12201-0509
}

Ribosomes conduct mRNA-directed synthesis of proteins according to the same principles in all organisms. In general, translation is accomplished in four steps: initiation, elongation, termination, and recycling. Termination takes place when a stop codon (UAA/UAG/UGA) is translocated into the decoding center of the ribosome. With the aid of a class-1 release factor (RF1 or RF2 in eubacteria, and eRF1 in eukaryotes), the finished peptide is released from the ribosome. In eubacteria, RF1 recognizes the stop codons UAA/UAG and RF2 recognizes UAA/UGA. In eukaryotes, a single class-1 peptide release factor, eRF1, recognizes all three stop codons.

A recent cryo-EM study of RF2 on the ribosome revealed an open conformation of the release factor, that is quite different from its compact X-ray structure [1]. This open conformation results in simultaneous interactions of RF2's two conserved structural motifs (SPF and GGQ) with the decoding center and the peptidyl-transferase center (PTC), respectively. Here, we present a cryo-EM study of the wild-type RF1 and a $\mathrm{GGQ} \rightarrow \mathrm{GAQ}$ mutant of RF1 in post- and pre-termination complexes with the $E$. coli $70 \mathrm{~S}$ ribosome, prepared in a highly efficient in vitro system [1]. The cryo-EM density attributable to RF1 reveals an open conformation on the ribosome, again in contrast to its compact X-ray structure [2]. Despite of the large difference between the X-ray structures of RF1 and RF2 in the constellation of domains, the models inferred from a flexible fitting of domains into the cryo-EM density maps are strikingly similar. However, despite this overall similarity, there are also significant differences in the interactions of the RFs with the GTP-associated center (GAC), as well as in the magnitude of the conformational change that brings each of the factors from the crystal to the ribosome structure. These observations suggest a structural basis for the different modes of interaction between the two release factors and the ribosome, in line with previous biochemical and kinetic data [3].

[1] U.B.S. Rawat et al., Nature 421 (2003) 87.

[2] D.H. Shin et al., J. Mol. Biol. 341 (2004) 227.

[3] This work was supported by HHMI and NIH grants R37 GM29169 and R01

GM55440 (to J.F.) and by the Swedish Research Council and the Swedish Foundation for Strategic Research (To M.E.). 


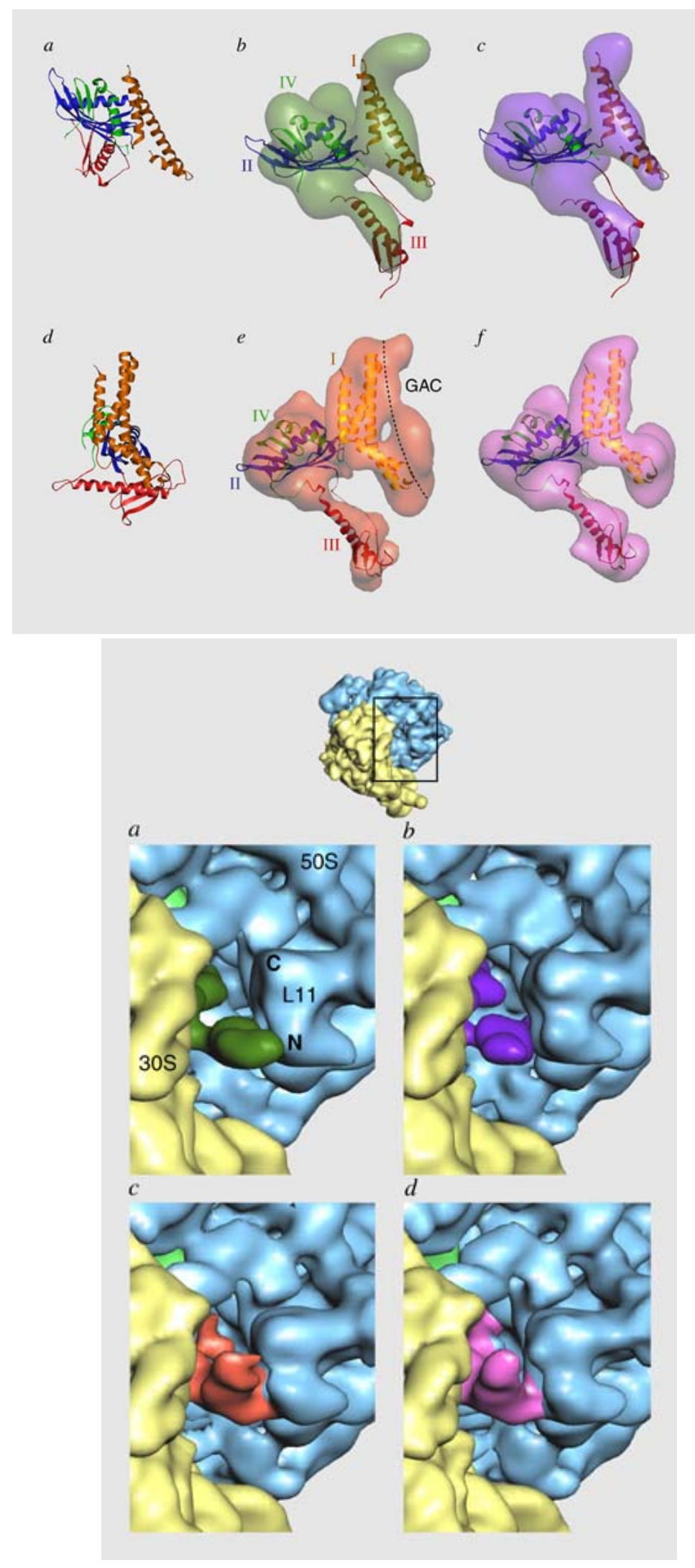

Figure 1. Comparison of RF1 and RF2 in the closed (X-ray structure) and open (ribosome-bound) conformations.

(a) X-ray structure of Thermotoga maritima RF1. (b, c) Fitting of (a) into the cryo-EM density maps of RF1 (GAQ) and RF1 (wt), shown in semitransparent green and purple, respectively. (d) X-ray structure of E. coli RF2. (e, f) Fitting of (d) into the cryo-EM density maps of RF2 (GAQ) and RF2 (wt) shown in semitransparent orange and magenta, respectively.

Figure 2. Interaction of RC with RFs. $(\mathrm{a}, \mathrm{b})$ Interaction of RF1 (GAQ) and RF1 (wt), with the ribosome. (c,d) Interaction of RF2 (GAQ) and RF2 (wt) with the ribosome. The ribosome is shown in a side view. 30S subunit (yellow), 50S subunit (blue).

Annotations:

L11, protein L11; C, C-terminal of L11; $\mathrm{N}, \mathrm{N}$-terminal of L11. 